Discussion Such scientific opinions on the quality, safety and efficacy of the medicines are provided by the EMA's Committee for Medicinal Products for Human Use (CHMP). Prior to this, it is recommended to agree on the data to be generated through scientific advice. The opinions are based on the same standards as used for those approved for Europe, with considerations for local conditions of use. To promote reliance on EMA scientific outputs and awareness of the procedure, two training events with regulators from Southern and from Western Africa are organised in partnership with WHO, NEPAD and local regulators in June 2018.

Conclusion We have shown that this 'article 58' procedure has a true impact and we encourage applications by companies developing medicines, aimed to prevent or treat diseases of significant public health interest, to be marketed outside the EU. This will ensure timely access of medicines by patients in target countries all over the world.

\section{OC 8432 EVALUATION OF AN ANTIBODY-DETECTING POINT-OF- CARE TEST FOR THE DIAGNOSIS OF TAENIA SOLIUM TAENIASIS AND NEUROCYSTICERCOSIS/CYSTICERCOSIS IN AN ENDEMIC AREA}

${ }^{1}$ Chishimba Mubanga* ${ }^{1}$ Kabemba Mwape, ${ }^{2}$ Gideon Zulu, ${ }^{1}$ Isaac Phiri, ${ }^{3}$ Chiara Trevisan ${ }^{3}$ Pierre Dorny, ${ }^{4}$ Sarah Gabriel, ${ }^{4}$ Inge Van Damme. ${ }^{1}$ University of Zambia, Lusaka, Zambia; ${ }^{2}$ Ministry of Health, Lusaka, Zambia; ${ }^{3}$ Institute of Tropical Medicine, Antwerp, Belgium; ${ }^{4}$ University of Ghent, Belgium

\subsection{6/bmjgh-2019-EDC.13}

Background Taenia solium taeniasis/(neuro)cysticercosis is a neglected parasitic zoonosis with significant economic and public health impacts. Neurocysticercosis is responsible for $30 \%$ cases of acquired epilepsy in endemic areas. Diagnosis and case management of neurocysticercosis/taeniasis in resource-limited endemic countries is challenging. Reliable, inexpensive and easy to use diagnostic tools with sufficient sensitivity and specificity are currently not available. A new point-of-care (POC) test based on recombinant rT24H and rES33 proteins developed by the Centre for Disease Control in Atlanta (US) which combines diagnosis of taeniasis and cysticercosis has been developed, however, its performance at community level is not known. The aim of this study is therefore, to evaluate the diagnostic performance of this test in a community setting.

Methods The study site is Mtandaza community, Sinda district, Eastern Province of Zambia. The diagnostic accuracy is being evaluated for taeniasis and (neuro) cysticercosis in 1200 randomly selected participants in a community-based study. The performance characteristics (sensitivity and specificity) for neurocysticercosis will be computed by cross-tabulating of POC results with those of the 'neurocysticercosis diagnosis' while a Bayesian approach will be used for cysticercosis and taeniasis to compare the performance of the index test with reference tests (enzyme-linked immuno-electrotransfer blot (EITB), B158/ B60 Ag-ELISA, Ab-ELISA, Copro-Ag ELISA, PCR).

Results Preliminary results of 505 POC tests so far conducted show that $0.8 \%$ were positive for taeniasis, 9.1\% for cysticercosis and, $4.6 \%$ were invalid or unclear. Except for Copro-Ag and B158/B60 Ag-ELISA for taeniasis and cysticercosis respectively, reference tests are yet to be conducted.

Conclusion Results will show the diagnostic value of the POC test and its applicability for use at community level in endemic areas. If successful, implementation of the tool will enable early detection of taeniasis and suspected neurocysticercosis cases and lead to improved patient management and treatment outcomes.

\section{OC 8435 MULTI-BIOMARKER TEST STRIP FOR POINT-OF-CARE SCREENING FOR ACTIVE TUBERCULOSIS: A FIVE- COUNTRY MULTI-CENTRE TEST EVALUATION}

${ }^{1}$ Paul Corstjens*, ${ }^{1}$ Anouk Van Hooij, ${ }^{1}$ Elisa Tjon Kon Fat, ${ }^{1}$ Shannon Herdigein, ${ }^{5}$ Anna Ritah Namuganga, ${ }^{6}$ Azaria Diergaardt, ${ }^{2}$ Hygon Mutavhatsindi, ${ }^{3}$ Awa Gindeh, ${ }^{4}$ Adane Mihret, ${ }^{2}$ Gian Van De Spuy, ${ }^{6}$ Gunar Gunther, ${ }^{4}$ Rawleigh Howe, ${ }^{5}$ Harriet MayanjaKizza, ${ }^{3}$ Jayne Sutherland, ${ }^{2}$ Novel N Chegou, ${ }^{1}$ Tom Ottenhoff, ${ }^{2}$ Gerhard Walzl, ${ }^{1}$ Annemieke Geluk. 'Leiden University Medical Center, The Netherlands; ${ }^{2}$ Stellenbosch University, South Africa; ${ }^{3}$ Medical Research Council, Serrekunda, The Gambia; ${ }^{4}$ Armauer Hansen Research Institute, Addis Ababa, Ethiopia; ${ }^{5}$ Makerere University School of Medicine, Kampala, Uganda; ${ }^{6}$ University of Namibia School of Medicine, Windhoek, Namibia

\subsection{6/bmjgh-2019-EDC.14}

Background Inexpensive rapid screening tests that can be used at the point-of-care (POC) are vital to combat tuberculosis. Particularly, less invasive non-sputum-based biomarker tests for all TB forms can help controlling transmission. Availability of such tests would significantly accelerate and streamline diagnostic approaches, improve cost-efficiency and decrease unnecessary costly GeneXpert referrals.

Methods Multi-biomarker test (MBT) devices measuring levels of selections of up to six serum proteins simultaneously on a single lateral flow (LF) strip were produced. The strip contains individual capture lines for a biomarker selection allowing discrimination of TB-patients from other respiratory diseases (ORD). Only biomarkers successfully evaluated with singleplex strips (single biomarker tests) were applied to the MBT device. Quantitative signals are recorded with a low-cost handheld reader compatible with the applied luminescent upconverting particle (UCP) label. Biomarker selection and algorithms used to distinguish potential-TB and ORD are flexible. Results Results obtained with MBT strips containing multiple test lines correlate well with singleplex LF strips. Using LF tests for 5 selected biomarkers a sensitivity of $94 \%$ and specificity of $96 \%$ could be achieved with a confirmed South African selection of $20 \mathrm{~TB}$ and 31 non-TB samples. Patients were designated TB positive when scoring a value above the cut-off threshold for at least 3 out of 5 biomarkers. Serum samples of potential TB patients collected at five medical research institutes (Ethiopia, Namibia, South Africa, The Gambia, Uganda) were tested locally with MBT strips comprised of CRP, SAA, IP-10, Ferritin, ApoA-I and IL-6 and results analysed to obtain an overall pan-Africa applicable signature.

Conclusion Evaluated POC applicable UCP-LF devices detecting serum biomarker signatures can help to distinguish active TB from other respiratory diseases and as such can prioritise highest-risk patients for further care. Ongoing prospective studies evaluate the MBT strip with fingerstick blood and do not require a laboratory or trained phlebotomist anymore.

\section{OC 8450 ABSENCE OF MINORITY HIV-1 DRUG-RESISTANT VARIANTS FOLLOWING MOTHER-TO-CHILD TRANSMISSION DOES NOT PREDICT VIROLOGIC SUCCESS OF FIRST-LINE ANTIRETROVIRAL THERAPY}

\footnotetext{
${ }^{1}$ Cissy Kityo* ${ }^{2,3}$ Tobias Rinke De Wit, ${ }^{1}$ Immaculate Nankya, ${ }^{4}$ Sheilla Balinda, ${ }^{2,5}$ Kim Sigaloff, ${ }^{1}$ Emmanuel Ndashimye, ${ }^{1}$ Peter Mugyenyi, ${ }^{6,7}$ Miguel Quinones-Mateu. ${ }^{1}$ Joint Clinical Research Centre, Kampala, Uganda; ${ }^{2}$ Amsterdam Institute for Global Health and Development, The Netherlands; ${ }^{3}$ Academic Medical Center, University of Amsterdam, The Netherlands; ${ }^{4}$ MRC/UVRI and LSHTM Uganda Research Unit, Uganda; ${ }^{5}$ Leiden University Medical Center, The Netherlands; ${ }^{6}$ Case Western Reserve University, Cleveland, USA; ${ }^{7}$ University Hospitals Cleveland Medical Center, USA
} 
Background Although minority HIV-1 drug-resistant HIV-1 variants may be selected under antiretroviral pressure, leading to therapy failure, their clinical significance remains controversial. This is particularly relevant in the case of prevention of mother-to-child transmission (MTCT), where transmitted drug resistance can affect treatment outcomes.

Methods An ultrasensitive HIV-1 genotyping assay based on deep sequencing (DEEPGENHIV) with a $1 \%$ mutation frequency sensitivity, was used to quantify MTCT drug-resistant variants in 38 prenatally HIV-infected children experiencing (Group I, $n=27$ ) or not (Group II, $n=11$ ) virologic failure 12 months after initiating first-line antiretroviral therapy (ART) as part of a paediatric cohort in Uganda.

Results Infants were infected with subtype $A(n=20), D(n=16)$ or $\mathrm{C}(\mathrm{n}=2)$ HIV-1 strains, distributed equally between both patients' groups. Similarly, no significant difference was observed in intra-patient HIV-1 diversity among viruses obtained from Group I or II individuals at baseline. DEEPGENHIV was able to detect all the mutations originally detected in samples obtained from four control patients in Group II, where drug resistance was identified at baseline using Sanger sequencing, e.g. K65R (78\% mutation frequency), K103N (47\%), or M184V (85\%). More importantly, a series of low abundance ( $<20 \%$ detection limit of Sanger) primary and compensatory mutations associated with resistance to PIs (D30N, Q48V), NRTIs (D67N, K219Q), or NNRTIs (L100I, $\mathrm{K} 103 \mathrm{~N})$ were identified in both groups of patients, although just a few seem to have been selected and became majority variants after 12 or 24 months of ART.

Conclusion DEEPGENHIV improves the detection of minority viral variants in infants following MTCT; however, most of the emergent HIV-1 drug resistance mutations were not present at low frequency at baseline in subjects failing ART, most likely being generated and selected following exposure to treatment. Further studies, using this or other ultrasensitive assays, are needed to better understand the transmission, dynamics and overall evolution of minority drug-resistant viruses in MTCT.

\section{OC 8453 SCALING-UP MASS TESTING AND TREATMENT EFFECTIVELY REDUCED MALARIA ASYMPTOMATIC PARASITAEMIA IN CHILDREN UNDER 15 YEARS OF AGE IN GHANA}

${ }^{1}$ Ignatius Cheng Ndong* ${ }^{1}$ Collins Stephan Ahorlu, ${ }^{1}$ Daniel Okyere ${ }^{1}$ Alexander A Nyarko, ${ }^{2}$ Alfred Amambua-Ngwa, 'Kwakwo A Koram. 'Noguchi Memorial Institute for Medical Research, University of Ghana, Accra, Ghana; ${ }^{2}$ Medical Research Council, Serrekunda, The Gambia

\subsection{6/bmjgh-2019-EDC.16}

Background Global efforts to scale up malaria control interventions that work, are gaining momentum. These include the use of long-lasting insecticide nets, intermittent preventive treatment and test, treat and track using artemisinin-based combination therapies. Mass testing, treatment and tracking (MTTT) of the entire populations complemented by homebased management (HBM) using volunteers could be an effective approach at lower cost. MTTT of children in Ghana has demonstrated a parasite load reduction of 90\%. However, unanswered questions include - Could this be scaled-up? What proportion of the community could be covered? What do we need? Can MTTT reduce asymptomatic parasitaemia levels in under-15 children?
Methods The target population for this study was 5000 asymptomatic individuals in seven communities in the Pakro subdistrict in Ghana. A community register was developed following a census. Community volunteers conducted quarterly house-to-house testing (using RDTs) and treating positive cases with ACTs. Between interventions HBM was conducted.

Results In those tested, asymptomatic malaria parasitaemia reduced from 1795 (36.3\%) in July 2017 to 942 (23.1\%) in March 2018. In eight months, parasitaemia declined by $43.5 \%$ and $37.3 \%$ in children under 15 and under 5, respectively. Coverage was $98.8 \%$ in July 2017 and $81.4 \%$ in March 2018. One of the challenges that surfaced was the fact that decrease in hospital attendance had a negative effect on money generated by the health facility. The district and subdistrict services, though appreciating the work, indicated that the negative effect on the health system may be serious and that measures need to be taken to address alternative financing for the health system.

Conclusion This study has demonstrated that combining MTTT and home-based management of malaria could reduce prevalence in under-15 children and that using community volunteers could ensure effective coverage at lower cost. There is need to start looking at financing of the health system without malaria.

\section{OC 8459 ASSESSMENT OF PARASITE CLEARANCE AFTER REPEATED TREATMENT WITH ARTESUNATE AMODIAQUINE, DIHYDROARTEMISININ-PIPERAQUINE, PYRONARIDINE-ARTESUNATE IN MALARIA PATIENTS IN BURKINA FASO}

Issiaka Soulama*, Sodiomon B Sirima. Centre National de Recherche et de Formation sur le Paludisme, Ouagadougou, Burkina Faso

\subsection{6/bmjgh-2019-EDC.17}

Background Reports from Southeast Asia showed delayed parasite clearance after treatment with known artemisinin-based combination therapies (ACTs), the first-line treatment for malaria. We then carried out a study in the framework of the WANECAM clinical trial to assess comparatively the parasite clearance time and rate from $P$. falciparum malaria patients repeatedly treated with the artesunate-amodiaquine (ASAQ), dihydroartemisinin-piperaquine (DHA-PQ) and artesunate-pyronaridine (PYR).

Methods A randomised, phase III/IV comparative, multicentre, open-label, parallel 3-arms trial was conducted in Banfora Health District area comparing the efficacy of a three-day regimen of DHA-PQ, PYR with ASAQ for the treatment of children (above 6 months) and adults with uncomplicated falciparum malaria. From August 2012 to December 2013, each randomised patient was followed up for 42 days over a period of two years. Treatment was directly observed, and blood smear samples were collected twice daily (12 hour \pm 2 hour) until parasite clearance.

The endpoints of the present sub-study were parasite clearance rate and time. The secondary endpoints included PCRcorrected and uncorrected cure rates.

Results Out of 2843 screened patients, 763 were recruited for parasite clearance endpoint analyses. The median parasite clearance time (PCT) was 24.1 hour (2-sided 95\% CI, 24.0 to 24.2 hour), 23.9 hour (2-sided 95\% CI, 23.8 to 24.0 hour) and 24.2 hour (2-sided 95\% CI, 24.1 to 24.5 hour), in PYR 